\title{
Difficulties in Correction of Recurrent Hypoglycemia in a Patient With a Progressive Course of Malignant Metastatic Insulinoma
}

\author{
Oksana V. Tsygankovaa, b, Antonina G. Antipenko ${ }^{\mathrm{b}}$, Natalia E. Evdokimova ${ }^{\mathrm{b}, \mathrm{d}}$, \\ Ludmila D. Latyntseva $^{b}$, Sabina S. Bairamova ${ }^{\text {b, }}$, Tatiana I. Batluk ${ }^{b}$
}

\begin{abstract}
This article describes a clinical observation of a patient with a progressive course of malignant metastatic insulinoma, despite repeated embolization and chemoembolization (CE) of the right and left hepatic arteries with doxorubicin and gemcitabine, with metastatic liver lesion and subsequent increase in the size of secondary foci. The key clinical problem was severe recurrent hypoglycemia, which persisted against the background of massive intravenous (IV) glucose infusions, shortacting somatostatin therapy, and subcutaneous (SC) glucagon injections, which not only was an impediment to the pathogenic cytostatic therapy but also has led to the fatal outcome of the patient.
\end{abstract}

Keywords: Severe hypoglycemia; Malignant insulinoma; Pancreatic neuroendocrine tumors; Case report; Chemoembolization of the hepatic arteries; Metastatic liver lesion; Octreotide; Glucagon

\section{Introduction}

Insulinomas are quite rare tumors, occurring with a frequency of 1 - 4 (cases) per million people per year, but are the most common among pancreatic neuroendocrine tumors $[1,2]$. In a benign process, surgical treatment in most cases contributes to complete remission of the disease (relapse is no more than

Manuscript submitted July 14, 2020, accepted July 17, 2020

Published online October 16, 2020

aNovosibirsk State Medical University, 52 Krasny Prospect Str., Novosibirsk 630091, Russia

${ }^{b}$ Research Institute of Therapy and Preventive Medicine-Federal Research Center Institute of Cytology and Genetics, Siberian Branch of Russian Academy of Sciences, 175/1 B. Bogatkov Str., Novosibirsk 630089, Russia

${ }^{\mathrm{c}}$ State Budgetary Healthcare Institution of the Novosibirsk Region (City Clinical Hospital of Emergency Medical Care No. 2), 41 Yakusheva Str., Novosibirsk 630102, Russia

${ }^{\mathrm{d} C}$ Corresponding Author: Natalia E. Evdokimova, Research Institute of Therapy and Preventive Medicine-Federal Research Center Institute of Cytology and Genetics, Siberian Branch of Russian Academy of Sciences, 175/1 B. Bogatkov Str., Novosibirsk 630089, Russia. Email: evdokimova1735.nsk@gmail.com

doi: https://doi.org/10.14740/jem666
$3 \%$ ); in the case of a malignant type of insulin-producing tumor in $2 / 3$ of cases metastasis is not observed [3]. At the same time, in case of the metastatic liver lesion, the average survival rate of patients does not exceed several years [4]. The diagnosis of malignant insulinoma in our patient was obvious and established more than 10 years ago and the features of the clinical picture, which deserve the attention of internists and endocrinologists, first of all, were determined by difficulties in correcting of severe recurrent hypoglycemia against the background of a steady progression of the underlying disease despite the polychemotherapy (PCT) and sevenfold appliance of high-tech care, embolization and chemoembolization (CE) of the hepatic arteries. The patient underwent complex symptomatic therapy, and a venous port system was installed, providing rapid glucose administration; however, death occurred during the course of another severe hypoglycemic attack.

\section{Case Report}

A 56-year-old woman for emergency indications was taken to the admission department of hospital on March 25, 2019 after amelioration of the severe hypoglycemia in the prehospital phase. She complained of feeling "blurred", excessive sweat, general weakness, shortness of breath with minimal physical activity, persistent nausea, occasional vomiting, diarrhea 2 - 3 times a day, and abdominal pain that worsens after eating.

From the anamnesis, it is known that at the beginning of April 2009, during the examination for severe hypoglycemia, a mass lesion of the pancreas with a size of $4.5 \times 2.6 \times 3.2$ $\mathrm{cm}$ was detected, involving splenic vessels and regional lymph nodes. On the April 28, 2009, she underwent operative treatment in the amount of the pancreas body and tail resection in combination with splenectomy and lymphadenectomy. The histological analysis confirmed the malignant neuroendocrine tumor of the pancreas: T2N1M0 IIB stage insulinoma. Later on, therapy with an intramuscular (IM) octreotide depot at a dose of $20 \mathrm{mg}$ once in 4 weeks was initiated, which she received for 1 year and with a positive effect.

In 2012 episodes of hypoglycemia resumed, with increasing frequency. During the control contrast-enhanced multispiral computed tomography of the abdomen on September 10, 2012, there were detected multiple metastatic foci in the liver up to $1.8 \mathrm{~cm}$, and in retroperitoneal lymphatic nodes. Therapy 
with octreotide depot was resumed successfully at the previous dose.

Worsening of patient condition repeated in spring 2016. During contrast-enhanced magnetic resonance imaging (MRI) of the abdomen, there was an increase of metastatic foci in the liver up to $10-14 \%$. The patient underwent one course of PCT according to the EC scheme (etoposide $100 \mathrm{mg} / \mathrm{m}^{2}$ intravenous (IV) in $1-3$ days + cisplatin $75 \mathrm{mg} / \mathrm{m}^{2} \mathrm{IV}$ on the first day once every 3 weeks). Subsequent courses of PCT were not possible due to the development of moderate anemia, which was not amenable to medication correction, and an increase of thrombocytosis up to $700 \times 10^{9} / \mathrm{L}$. As an alternative treatment, she received interferon-alpha therapy (three million IU three times a week SC). The dose of octreotide depot was increased to 30 mg every 4 weeks. Against this background, the frequency of hypoglycemia had decreased to 2 - 3 episodes per week, and severe hypoglycemia to $1-2$ per month. During that period, along with low values of plasma glucose, its increase to $11-12$ $\mathrm{mmol} / \mathrm{L}$ was registered. The patient repeatedly underwent $\mathrm{CE}$ of right and left hepatic arteries with doxorubicin and gemcitabine (August 2017, December 2017, February 2018, and March 2018).

The patient's condition has gradually deteriorated since January 2019. Despite the observance of a proper fractional diet, regular injections of octreotide depot at an increased dose of $40 \mathrm{mg}$ every 4 weeks under slight delays in food intake, there were attacks of hypoglycemia to 3 - 4 times per day with a rapid increase in symptoms, and the clinical picture of neuroglycopenia up to the loss of consciousness. The minimal values of plasma glucose at that period were $1.8 \mathrm{mmol} / \mathrm{L}$. Later on, the food intake acquired a forced character as it was accompanied by an aversion to food, nausea, and vomiting of food eaten; there was an unintended weight reduction by $3 \mathrm{~kg}$ in 2 months, as well as deterioration of short-term memory, increased negativity, and irritability.

According to the data of contrast-enhanced abdomen MRI on February 08, 2019, there was detected negative dynamics in the form of an increase in the size of metastases in the liver, lymphadenopathy of hepatic hilum. In March 2019, an oncologist consulted the patient, and recommended embolization of the right hepatic artery followed by CE of the left hepatic artery as the second stage; however, due to the recurrent hypoglycemia up to 10 times per day, the patient was intolerant to any medical manipulations, for which reason she was hospitalized in the therapeutic department for stabilization of her condition.

The patient had concomitant pathology: primary hypothyroidism, corrected by daily intake of levothyroxine $25 \mu \mathrm{g}$ (thyroid-stimulating hormone $3.2 \mathrm{mU} / \mathrm{L}$ from February 13, 2019); nephropathy (drug-induced, paraneoplastic), chronic kidney disease G3aA2; secondary moderate anemia of complex genesis; chronic gastritis (not in exacerbation); duodenogastric bile reflux; peptic ulcer disease of the duodenum with cicatricial deformation of the bulb. There was no significant family history for cancer, endocrinopathy, including components of multiple endocrine neoplasia type 1 (MEN-1) syndrome. The patient had one childbirth with menopause at 45 years.

During the physical examination, the general condition was severe. Body mass index was $22.2 \mathrm{~kg} / \mathrm{m}^{2}$.

The skin of the upper half of the trunk and upper limbs was hyperemic, with a cyanotic tinge, moderately moist. The liver under palpation was tuberous, and there was determined a dense moderately painful mass with a diameter up to $8 \mathrm{~cm}$ in the projection of the liver and the head of the pancreas.

Labs showed hemoglobin $87 \mathrm{~g} / \mathrm{L}$, serum iron $3.1 \mu \mathrm{mol} / \mathrm{L}$ (10.5 - $32.2 \mu \mathrm{mol} / \mathrm{L})$, albumin $32.7 \mathrm{~g} / \mathrm{L}$ (35 - $52 \mathrm{~g} / \mathrm{L})$, aspartate aminotransferase $54.6 \mathrm{U} / \mathrm{L}(<39 \mathrm{U} / \mathrm{L})$, alanine aminotransferase $85 \mathrm{U} / \mathrm{L}(<32 \mathrm{U} / \mathrm{L})$, gamma-glutamyltransferase 379.8 $\mathrm{U} / \mathrm{L}(7-38 \mathrm{U} / \mathrm{L})$, alkaline phosphatase $867.7 \mathrm{U} / \mathrm{L}$ (30 - 120 $\mathrm{U} / \mathrm{L}), \mathrm{Na} 135 \mathrm{mmol} / \mathrm{L}(136$ - $145 \mathrm{mmol} / \mathrm{L}), \mathrm{Ca} 2.15 \mu \mathrm{mol} / \mathrm{L}$ (2.2 - $2.65 \mu \mathrm{mol} / \mathrm{L}), \mathrm{K} 4.9 \mathrm{mmol} / \mathrm{L}$ (3.7 - $5.2 \mathrm{mmol} / \mathrm{L})$, Chronic Kidney Disease Epidemiology Collaboration (CKD-EPI) creatinine equation $52 \mathrm{~mL} / \mathrm{min} / 1.73 \mathrm{~m}^{2}$, total cholesterol 2.5 $\mathrm{mmol} / \mathrm{L}$, moderate hypocoagulation, insulin $83.6 \mu \mathrm{IU} / \mathrm{mL}(2.7$ -10.4 $\mu \mathrm{U} / \mathrm{mL})$, C-peptide $14.274 \mathrm{nmol} / \mathrm{L}$ (0.26 - $1.73 \mathrm{nmol} / \mathrm{L})$ with glycemia $5.3 \mathrm{mmol} / \mathrm{L}$, the minimum registered value of glycemia $1.1 \mathrm{mmol} / \mathrm{L}$, index of insulin $(\mu \mathrm{IU} / \mathrm{mL}) / \mathrm{glucose}(\mathrm{mg} /$ dL) $0.88(<0.3)$, glycated hemoglobin for a month before hospitalization was $6.6 \%$, during it $4.4 \%$, and proteinuria $0.3 \mathrm{~g} / \mathrm{L}$.

Electrocardiogram, chest X-ray, and ultrasound of the kidneys and adrenal glands were unremarkable.

As for the treatment, at the time of the hospital stay, the patient received octreotide depot $40 \mathrm{mg}$ every 4 weeks. Additionally, during the first 4 days of hospital stay, she received IV infusions of 5\% glucose solution in the volume of $500 \mathrm{~mL}$ three times a day as well as fractional IV bolus injections 40 $\mathrm{mL} 40 \%$ glucose solution three times a day (total $123 \mathrm{~g}$ of glucose/day). Taking into account the remaining hypoglycemia up to $1.1 \mathrm{mmol} / \mathrm{L}$, it was decided to perform continuous IV infusion of $40 \%$ glucose solution by infusion pump along with bolus situational injections. The dose of the drug was calculated based on the values of glycemia and ranged from 72 to $216 \mathrm{~g} /$ day. Despite this, keeping the euglycemic corridor was not possible, and low values of glycemia up to $2.1 \mathrm{mmol} / \mathrm{L}$ were recorded, which was accompanied by the clinical picture of neuroglycopenia, loss of consciousness, during the whole period of hospital stay monitoring of glucose. Figure 1 shows the dynamics of the fingerstick glucose level against the background of permanent infusions of $40 \%$ glucose solution by infusion pump at the rate of $10-15 \mathrm{~mL} / \mathrm{h}$ and additional bolus injections $40-60 \mathrm{~mL}$ three times a day.

In this connection, the therapy regimen was enhanced with short-acting octreotide, injected SC at a dose of $100 \mu \mathrm{g}$ three times a day, and dexamethasone $8 \mathrm{mg}$ IV three times a day. Also, $1 \mathrm{mg}$ of glucagon solution was administered in a situationally fractional manner; however, the effect of the drug administration was not observed.

Complex therapy included $0.9 \%$ saline solution 200 - 400 $\mathrm{mL}$ /day to correct hyponatremia due to loss of electrolytes during vomiting, forced diuresis; also, taking into account hypocalcemia she received calcium injections. Considering the massive infusion therapy, injections of high-osmolar glucose solutions, recurrent hypoglycemia, in agreement with resuscitators, the patient was prescribed low doses of diuretics (furosemide 40 $\mathrm{mg}$ in the morning and spironolactone $25 \mathrm{mg}$ in the morning and afternoon) to avoid brain edema. The patient also was prescribed ursodeoxycholic acid $250 \mathrm{mg}$ three times a day to correct cytolysis and cholestasis syndromes, proton pump inhibitors to protect gastric mucosa and iron drugs to correct anemia. 


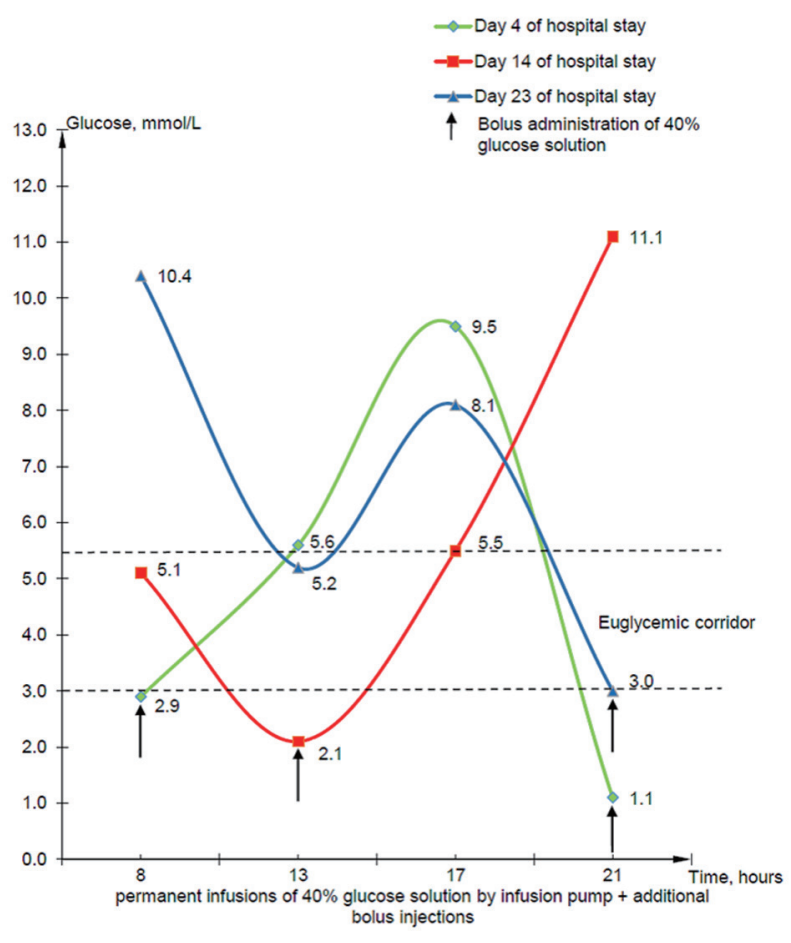

Figure 1. Dynamics of the fingerstick glucose level of patient at the beginning, in the middle, and at the end of hospitalization.

With account taken of multicomponent treatment provided, the maximum possible compensation of carbohydrate metabolism was achieved by keeping glycemia levels in the range of $3.0-10.4 \mathrm{mmol} / \mathrm{L}$, which allowed to embolize the hepatic artery with lipiodol and hemostatic sponge on the 24th day of hospitalization.

Also, in April 2019, taking into account continuing requirement for repeated glucose injections, a port system was implanted into the right subclavian vein, which ensured the patient tolerance for another course of CE of tumor vessels, the left hepatic artery (conducted on June 08, 2019). At the same time, due to recurrent hypoglycemic conditions, by the decision of the case conference, an attempt was made to reduce the dose of octreotide depot to $10 \mathrm{mg}$ once in 4 weeks IM in combination with ongoing therapy with interferon-alpha three million IU three times a week SC. Despite all the treatment, the patient has died in early July 2019 during severe hypoglycemia.

\section{Discussion}

In the case, the patient lived for 10 years, despite the progression of the oncological disease, on the back of ongoing chemotherapy, interferon therapy, high-tech care in the form of $\mathrm{CE}$ and embolization of different hepatic arteries, which she underwent seven times; however, she had an extremely low quality of life due to severe recurrent hypoglycemic syndrome. The diagnosis was made promptly, at the first presentation, which is not typical and is associated with a bright clinical picture due to the malignant nature of the tumor. Taking into account long- term endocrinologist supervision of woman and the absence of other components of MEN-1 syndrome, we can assume the sporadic nature of insulinoma $[5,6]$.

At the final stage of the disease, with the appearance of metastatic liver damage, the carcinoid syndrome (diarrhea, abdominal pain after eating, diffuse hyperhidrosis, and cyanosis of the skin) attached, which is probably due to a disturbance of serotonin degradation by the liver, along with other manifestations of hepatic cell failure (hypooncia, hypocoagulation, hypocholesterolemia), as well as its increased autonomous production [7].

After surgical treatment, $\mathrm{CE}$ and embolization of hepatic arteries led only to a short-term decrease in the frequency of hypoglycemia as insulin in large quantities continued to be secreted by the remaining functionally active metastases. During literature search we have encountered a description of a combination of malignant insulinoma with previously diagnosed type 2 diabetes. No more than 30 cases of such a combination have been described in the world literature from 1927 to the present time [8]. In that clinical observation, we also had a rare opportunity to observe a combination of two opposite in their essence and clinical picture conditions, hyperglycemia and insulinoma, with characteristic hypoglycemic symptoms. It should be assumed that hyperglycemia, appeared 3 years after resection of the tail and body of the pancreas, chronologically associated with an increase in the dose of octreotide depot from 20 to $30 \mathrm{mg}$, had the combined genesis, including pancreatogenic component and effects of the long-acting somatostatin analog. Cyclical insulin production by a malignant tumor on the one side and a decrease in beta-cell volume and pancreatic cancer, which, according to literature data, regardless of its size due to paracrine and autocrine effects, can lead to hyperglycemia, on the other side $[9,10]$, resulting in severe glycemic fluctuations.

Difficulties in correcting hypoglycemic syndrome, which determined the severity of the patient's condition and was the direct cause of her death, despite all the efforts made by doctors, should be emphasized separately. Supervision of patient was carried out under the conditions of the ordinary therapeutic department due to the lack of places in the intensive care unit and included multiple examinations during the day and night, measurement of whole blood glucose from the finger up to 20 times a day, the use of a wide array of available medications, including long-and short-acting somatostatin analogues, glucagon, glucocorticosteroids, constant infusion and fractional bolus administration of hypertonic glucose solution.

This approach made it possible to achieve maximum available compensation for glycemic levels and allowed to perform right hepatic artery embolization and to implant a port system for long-term infusion or bolus therapy to rapidly treat hypoglycemia, including severe one.

\section{Conclusions}

The case report illustrates that it is possible to achieve a relatively high life expectancy in the modern complex oncological approach, even with the malignant nature of insulinoma with hepatic metastasis. Moreover, hypoglycemic conditions may 
be dominant in the clinical picture, requiring competent supervision and integration of specialists, endocrinologists, and general practitioners.

\section{Acknowledgments}

None to declare.

\section{Financial Disclosure}

The study was partially performed in the framework of the budget theme of the state assignment number AAAAA17-117112850280-2.

\section{Conflict of Interest}

None to declare.

\section{Informed Consent}

The manuscript has been sufficiently de-identified to protect the patient. She has since died and is unable to provide informed consent.

\section{Author Contributions}

OVT and AGA contributed to the acquisition and interpretation of the data, drafting and critically revising the manuscript, approving the final publishable version of the manuscript, and agreeing to be accountable for all aspects of the work. NEE and LDL contributed to the acquisition of the data, drafting the manuscript, approving the publishable version of the manuscript, and agreeing to be accountable for all aspects of the work. TIB and SSB contributed to drafting the manuscript, approving the final publishable version of the manuscript, and agreeing to be accountable for all aspects of the work.

\section{Data Availability}

The authors declare that data supporting the findings of this manuscript are available within the article. Also, any inquiries regarding supporting data availability of this article should be directed to the corresponding author.

\section{References}

1. Zhuo F, Anastasopoulou C. Insulinoma. In: StatPearls. Treasure Island (FL), 2020.

2. Subasinghe D, Gunatilake SSC, Dassanyake VE, Garusinghe C, Ganewaththa E, Appuhamy C, Somasundaram NP, et al. Seeking the unseen: Localization and surgery for an occult sporadic insulinoma. Ann Hepatobiliary Pancreat Surg. 2020;24(2):234-238.

3. Demidova TYu, Titova VV. Long-standing relapse of insulinoma in clinical practice. RMJ. 2018;11(II):122-125. (In Russ.).

4. Ueda K, Taira T, Hakoda H, Nakata S, Okata S, Nagai T, Aoki S, et al. Giant insulinoma: report of a case and review of published reports. Surg Case Rep. 2016;2(1):136.

5. Falconi M, Eriksson B, Kaltsas G, Bartsch DK, Capdevila J, Caplin M, Kos-Kudla B, et al. ENETS consensus guidelines update for the management of patients with functional pancreatic neuroendocrine tumors and nonfunctional pancreatic neuroendocrine tumors. Neuroendocrinology. 2016;103(2):153-171.

6. Marx SJ. Recent topics around multiple endocrine neoplasia type 1. J Clin Endocrinol Metab. 2018;103(4):12961301.

7. Rubin de Celis Ferrari AC, Glasberg J, Riechelmann RP. Carcinoid syndrome: update on the pathophysiology and treatment. Clinics (Sao Paulo). 2018;73(suppl 1):e490s.

8. Podzolkov VI, Dragomiretskaya NA, Koroleva TV, Kavtaradze NN, Yakovleva NN, Podzolkov AV. A rare cause of hypoglycemia in an elderly patient with type 2 diabetes is malignant metastatic insulinoma. Klinicheskaya Meditsina. 2014;2:65-70. (In Russ.).

9. Gut P, Waligorska-Stachura J, Czarnywojtek A, SawickaGutaj N, Baczyk M, Ziemnicka K, Fischbach J, et al. Management of the hormonal syndrome of neuroendocrine tumors. Arch Med Sci. 2017;13(3):515-524.

10. Okuma H, Bouchi R, Masuda S, Takeuchi T, Murakami M, Minami I, Izumiyama H, et al. Suppression of extrapancreatic glucagon by octreotide may reduce the fasting and postprandial glucose levels in a diabetic patient after total pancreatectomy. Intern Med. 2017;56(22):30613066. 